\title{
CHARACTERIZATION OF TURBULENCE FROM SUBMILLIMETER DUST EMISSION
}

\author{
Shadi Chitsazzadeh ${ }^{1,2}$, Martin Houde ${ }^{2,3}$, Roger H. Hildebrand ${ }^{4,5}$, and John Vaillancourt ${ }^{6}$ \\ ${ }^{1}$ Department of Physics and Astronomy, The University of Victoria, Victoria, BC V8P 5C2, Canada \\ ${ }^{2}$ Department of Physics and Astronomy, The University of Western Ontario, London, ON N6A 3K7, Canada \\ ${ }^{3}$ Division of Physics, Mathematics and Astronomy, California Institute of Technology, Pasadena, CA 91125, USA \\ ${ }^{4}$ Department of Astronomy and Astrophysics and Enrico Fermi Institute, The University of Chicago, Chicago, IL 60637, USA \\ ${ }^{5}$ Department of Physics, The University of Chicago, Chicago, IL 60637, USA \\ ${ }^{6}$ Stratospheric Observatory for Infrared Astronomy, Universities Space Research Association, NASA Ames Research Center, Moffet Field, CA 94035, USA \\ Received 2011 November 19; accepted 2012 February 5; published 2012 March 20
}

\begin{abstract}
In this paper, we use our recent technique for estimating the turbulent component of the magnetic field to derive the structure functions of the unpolarized emission as well as that of the Stokes $Q$ and $U$ parameters of the polarized emission. The solutions for the structure functions to $350 \mu \mathrm{m}$ SHARP polarization data of OMC-1 allow the determination of the corresponding turbulent correlation length scales. The estimated values for these length scales are $9.4 \pm 0.1,7^{\prime \prime} .3 \pm 00^{\prime} .1,12^{\prime \prime} .6 \pm 0$ '. 2 (or $20.5 \pm 0.2,16.0 \pm 0.2$, and $27.5 \pm 0.4 \mathrm{mpc}$ at $450 \mathrm{pc}$, the adopted distance for OMC-1) for the Stokes $Q$ and $U$ parameters, and for the unpolarized emission $N$, respectively. Our current results for $Q$ and $U$ are consistent with previous results obtained through other methods and may indicate presence of anisotropy in magnetized turbulence. We infer a weak coupling between the dust component responsible for the unpolarized emission $N$ and the magnetic field $\boldsymbol{B}$ from the significant difference between their turbulent correlation length scales.
\end{abstract}

Key words: ISM: clouds - ISM: magnetic fields - polarization - turbulence

\section{INTRODUCTION}

Magnetic fields play a crucial role in the formation of stars through various processes, ranging from magnetic support against gravitational collapse (see the reviews by Shu et al. 1999 and Mouschovias \& Ciolek 1999) to magnetic braking (Nakano 1984). On the other hand, turbulence has also been suggested to be a regulating factor in the development of the star formation process (see the reviews by Mac Low \& Klessen 2004 and Elmegreen \& Scalo 2004). The relative importance of these two agents during the star formation process remains controversial (e.g., Mouschovias \& Tassis 2009; Crutcher et al. 2010).

The magnetic field threading a molecular cloud is composed of a large-scale ordered component and a turbulent (or random) one. Characterization of the latter, e.g., through dust polarization measurements, is highly desirable (e.g., Lai et al. 2001, 2002, 2003; Crutcher et al. 2004). For example, with this information one can use the method introduced by Chandrasekhar \& Fermi (1953) together with the estimates of gas density and line-ofsight velocity dispersion in molecular clouds (from appropriate molecular species, e.g., $\mathrm{H}^{13} \mathrm{CO}^{+}$; see Hildebrand et al. 2009 and Houde et al. 2009) to derive the strength of the plane of the sky component of the magnetic field in these regions (e.g., Houde 2004; Girart et al. 2006; Curran \& Chrysostomou 2007; Attard et al. 2009). It can also provide us with a measure of the turbulent energy content of the gas.

A potential problem in using such a method is the necessity to make assumptions on the structure of the large-scale component of the field. These assumed models for the morphology of the magnetic field, even though derived from a polarization map, may still lead to imprecise values for the turbulent component as the assumptions regarding the large scale will not follow its true structure. Hildebrand et al. (2009) introduced a new technique where the dispersion function of the polarization angle is used to estimate the turbulent component of the field without making any assumptions on the morphology of the large-scale magnetic field. This method has been studied in more details by Houde et al. (2009), where the effect of signal integration was taken into account in the evaluation of the ratio of the turbulent to largescale magnetic field strength. This has also allowed, among other things, the determination of the turbulent magnetic field correlation length scale.

In this paper, we will use the methods introduced in Houde et al. (2009) to compare the turbulent character of the polarized emission (emanating from the dust component that is coupled to the magnetic field) and that of the unpolarized emission by estimating their turbulent correlation length scales. We will start by deriving the cloud- and beam-integrated structure functions for the Stokes $Q$ and $U$ parameters and the unpolarized emission $N$ in Section 2. We will then proceed with presenting the solution for the structure functions assuming Gaussian profiles for the beam and the turbulent autocorrelation functions of $Q, U$, and $N$. In Section 3, we use the previously published $350 \mu \mathrm{m}$ SHARP polarization map of OMC-1 of Vaillancourt et al. (2008) to determine the turbulent correlation lengths, and then use these results to compare the turbulent character of the polarized emission and the unpolarized emission and gain information on the nature of the turbulence and magnetic field existing in the region of interest.

\section{ANALYSIS}

\subsection{The Cloud-and Beam-integrated Structure Function of the Polarized Components of the Emission}

The linear polarized emission is observationally determined through the measurements of the cloud- and beam-integrated Stokes parameters

$$
\begin{aligned}
& \bar{Q}(\mathbf{r})=\iint H(\mathbf{r}-\mathbf{a})\left[\frac{1}{\Delta} \int Q(\mathbf{a}, z) d z\right] d^{2} a \\
& \bar{U}(\mathbf{r})=\iint H(\mathbf{r}-\mathbf{a})\left[\frac{1}{\Delta} \int U(\mathbf{a}, z) d z\right] d^{2} a,
\end{aligned}
$$


with

$$
\bar{P}(\mathbf{r})=\sqrt{\bar{Q}^{2}(\mathbf{r})+\bar{U}^{2}(\mathbf{r})}
$$

the integrated polarized emission, $H(\mathbf{r})$ the beam profile, and $\Delta$ the maximum depth of the cloud along any line of sight (Houde et al. 2009). The unpolarized emission $N$ can be estimated through the measurements of the total emission $I$ and the polarized emission $P$ with

$$
N(\mathbf{r})=I(\mathbf{r})-P(\mathbf{r}) .
$$

In analogy to $\bar{Q}$ and $\bar{U}, \bar{N}$ is defined as

$$
\bar{N}(\mathbf{r})=\iint H(\mathbf{r}-\mathbf{a})\left[\frac{1}{\Delta} \int N(\mathbf{a}, z) d z\right] d^{2} a .
$$

The quantity for $\bar{N}(\mathbf{r})$ defined in Equation (5) is, however, not directly measurable. We therefore use the following approximation:

$$
\bar{N}(\mathbf{r}) \simeq \bar{I}(\mathbf{r})-\bar{P}(\mathbf{r})
$$

The two-dimensional integrals in Equations (1), (2), and (5) are over all space. The $z$-axis is along the line of sight (unit basis vector $\mathbf{e}_{z}$ ), and $\mathbf{r}$ is the two-dimensional polar radius vector on the plane of the sky (unit basis vector $\mathbf{e}_{r}$ ) such that

$$
\mathbf{x}=r \mathbf{e}_{r}+z \mathbf{e}_{z}
$$

Due to the mathematical similarity between the definitions of $\bar{Q}$, $\bar{U}$, and $\bar{N}$, the analyses will be shown for only one of them (the $\bar{Q}$ component), the results for $\bar{U}$ and $\bar{N}$ follow in a straightforward manner.

Similarly to the characterization of the magnetic field presented in Hildebrand et al. (2009) and Houde et al. (2009), we will divide the emission - either polarized or unpolarized - into ordered and turbulent components:

$$
Q(\mathbf{a}, z)=Q_{0}(\mathbf{a}, z)+Q_{t}(\mathbf{a}, z) .
$$

In order to gain a quantitative estimate of the turbulent component of the magnetic field in molecular clouds, it is necessary to make some assumptions about the Stokes parameters $(Q$ and $U)$ as well as the unpolarized emission $N$. More precisely, we assume statistical independence between the ordered and turbulent components. We will therefore have the following averages for any two points $\mathbf{x}$ and $\mathbf{y}$ :

$$
\begin{aligned}
\left\langle Q_{0}(\mathbf{x})\right\rangle & =Q_{0}(\mathbf{x}) \\
\left\langle Q_{t}(\mathbf{x})\right\rangle & =0 \\
\left\langle Q_{0}(\mathbf{x}) \cdot Q_{t}(\mathbf{y})\right\rangle & =\left\langle Q_{0}(\mathbf{x})\right\rangle \cdot\left\langle Q_{t}(\mathbf{y})\right\rangle=0 .
\end{aligned}
$$

Moreover, we assume that $Q$ is stationary and isotropic (see Equations (15) and (16) below). Note that the distance between the two points where the Stokes parameter $Q$ is measured is confined to the plane of the sky unless otherwise stated.

The autocorrelation function of $Q$ can be introduced as

$$
R_{Q}(v, u)=\left\langle Q(\mathbf{a}, z) Q\left(\mathbf{a}^{\prime}, z^{\prime}\right)\right\rangle,
$$

with $u=\left|z^{\prime}-z\right|$ and $v=\left|\mathbf{a}^{\prime}-\mathbf{a}\right|$. Due to the assumed statistical independence of the ordered and turbulent components, it is possible to decompose these parts of the autocorrelation function in the following way:

$$
R_{Q}(v, u)=R_{Q, 0}(v, u)+R_{Q, t}(v, u),
$$

with

$$
R_{Q, j}(v, u)=\left\langle Q_{j}(\mathbf{a}, z) Q_{j}\left(\mathbf{a}^{\prime}, z^{\prime}\right)\right\rangle
$$

in which $j$ stands for " 0 " or " $t$ " for the ordered and turbulent parts, respectively.

In order to estimate the structure function of the Stokes parameter, it is necessary to specify some characteristics of the autocorrelation function, as well as the telescope beam profile. Following Houde et al. (2009) we write

$$
R_{Q}(v, u)=R_{Q, 0}(v, u)+\left\langle Q_{t}^{2}\right\rangle e^{-\left(v^{2}+u^{2}\right) / 2 \delta_{Q}^{2}},
$$

with $\delta_{Q}$ the correlation length scale for the turbulent component of $Q$. For the time being, we assume that $U$ and $N$ have turbulent correlation length scales $\delta_{U}$ and $\delta_{N}$, which are potentially different from $\delta_{Q}$. Moreover, these correlation lengths are both assumed to be much smaller than the thickness of the cloud $(\Delta)$. The beam profile is also assumed Gaussian:

$$
H(r)=\frac{1}{2 \pi W^{2}} e^{-r^{2} / 2 W^{2}}
$$

with $W$ the beam radius.

For the structure function (Falceta-Gonçalves et al. 2008; Hildebrand et al. 2009) of $\bar{Q}$ we start with the following definition:

$$
\begin{aligned}
\left\langle\Delta \bar{Q}^{2}(\ell)\right\rangle & \equiv\left\langle[\bar{Q}(\mathbf{r})-\bar{Q}(\mathbf{r}+\ell)]^{2}\right\rangle \\
& =2\left[\left\langle\bar{Q}^{2}\right\rangle-\langle\bar{Q} \bar{Q}(\ell)\rangle\right],
\end{aligned}
$$

where we have used the aforementioned assumptions of isotropy and stationarity and

$$
\langle\bar{Q} \bar{Q}(\ell)\rangle \equiv\langle\bar{Q}(\mathbf{r}) \bar{Q}(\mathbf{r}+\ell)\rangle .
$$

Under these constraints, decomposing the ordered and turbulent components of $Q$, and incorporating the analytical solution provided in Appendix A of Houde et al. (2009) we can write

$$
\begin{aligned}
\langle\bar{Q} \bar{Q}(\ell)\rangle= & \frac{\langle\xi(\ell)\rangle}{2}+\sqrt{2 \pi}\left\langle Q_{t}^{2}\right\rangle\left[\frac{\delta_{Q}^{3}}{\left(\delta_{Q}^{2}+2 W^{2}\right) \Delta}\right] \\
& \times e^{-\ell^{2} / 2\left(\delta_{Q}^{2}+2 W^{2}\right)},
\end{aligned}
$$

where

$$
\begin{aligned}
\langle\xi(\ell)\rangle= & 2 \iiint \int H(\mathbf{a}) H\left(\mathbf{a}^{\prime}+\ell\right) \\
& \times\left[\frac{2}{\Delta} \int\left(1-\frac{u}{\Delta}\right) R_{Q, 0}(v, u) d u\right] d^{2} a^{\prime} d^{2} a .
\end{aligned}
$$

Inserting Equations (17) and (18) into Equation (15) we get

$$
\begin{aligned}
\left\langle\Delta \bar{Q}^{2}(\ell)\right\rangle= & {[\langle\xi(0)\rangle-\langle\xi(\ell)\rangle]+2 \sqrt{2 \pi}\left\langle Q_{t}^{2}\right\rangle } \\
& \times\left[\frac{\delta_{Q}^{3}}{\left(\delta_{Q}^{2}+2 W^{2}\right) \Delta}\right]\left[1-e^{-\ell^{2} / 2\left(\delta_{Q}^{2}+2 W^{2}\right)}\right] .
\end{aligned}
$$

The first term on the right-hand side of the above equation (within brackets) is solely due to the ordered component of the Stokes $Q$ parameter and not turbulence. This term can be expanded as a Taylor series (similarly to the analysis presented in the Appendix of Houde et al. 2009):

$$
\langle\xi(0)\rangle-\langle\xi(\ell)\rangle=\sum_{i=1}^{\infty} c_{2 i} \ell^{2 i},
$$



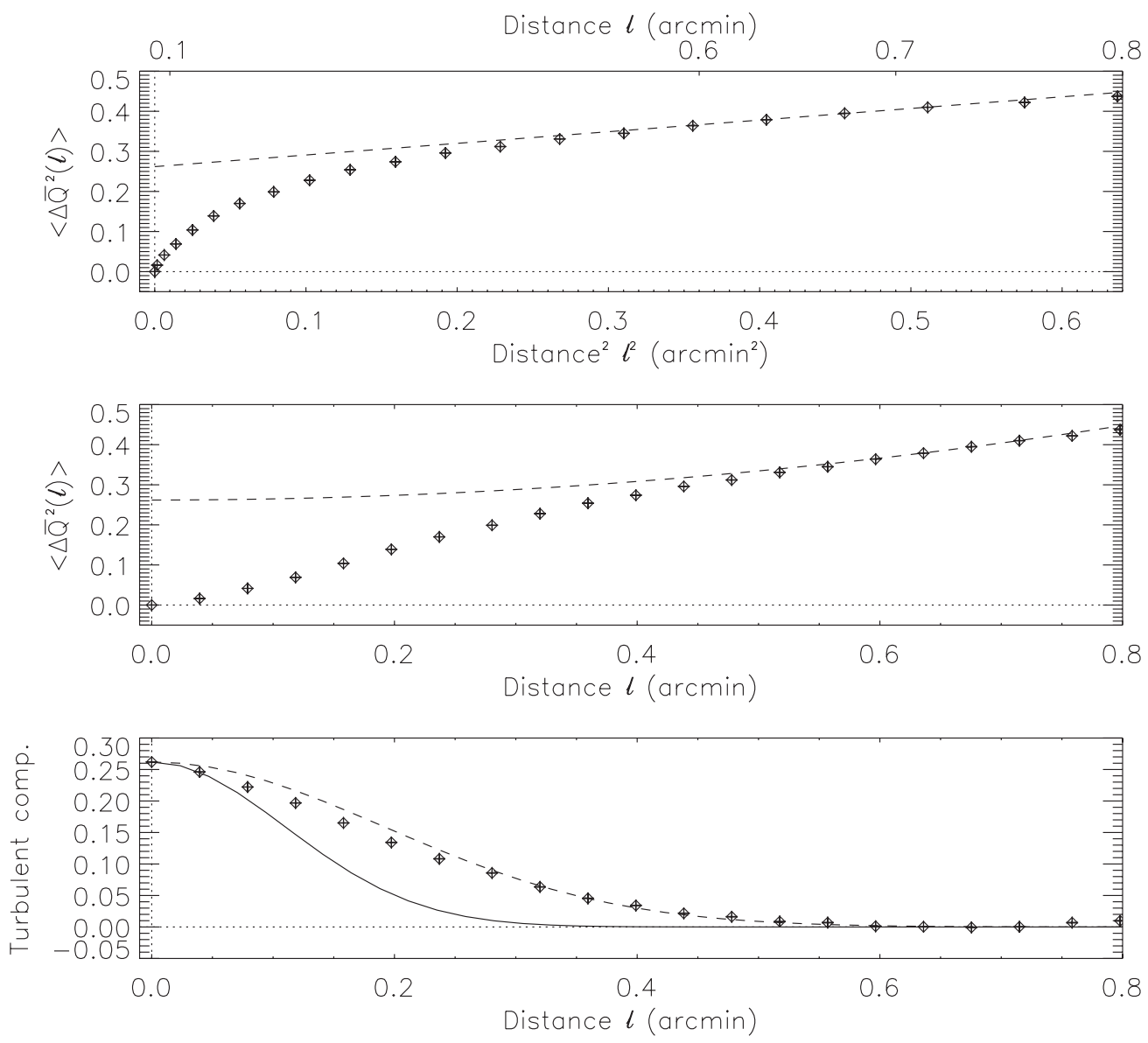

Figure 1. Structure function $\left\langle\Delta \bar{Q}^{2}(\ell)\right\rangle$ using the previously published $350 \mu \mathrm{m}$ SHARP polarization map of OMC-1 of Vaillancourt et al. (2008). Top: Equation (24) (broken curve) is fitted to the data (symbols) and plotted as a function of $\ell^{2}$; middle: similar to top but plotted as a function of $\ell$; bottom: the difference between the data points and the fit of Equation (24) is shown as symbols with the broken curve depicting the fit of Equation (23) with $\delta_{Q}=9$ !' $4 \pm 0$ '. 1 . The solid curve shows the contribution of the telescope beam alone (i.e., when $\delta_{Q}=0$ ). The telescope beam is assumed to be Gaussian.

while the isotropy in $\ell$ is incorporated by performing the summation only on terms of even orders in $\ell$. For small displacements satisfying $\ell \lesssim W$, the ordered term can be described adequately by keeping the first-order term in the Taylor series. Thus, when $\ell \lesssim W$ we will have

$$
\begin{aligned}
\left\langle\Delta \bar{Q}^{2}(\ell)\right\rangle \simeq & m_{Q}^{2} \ell^{2}+2 \sqrt{2 \pi}\left\langle Q_{t}^{2}\right\rangle\left[\frac{\delta_{Q}^{3}}{\left(\delta_{Q}^{2}+2 W^{2}\right) \Delta}\right] \\
& \times\left[1-e^{-\ell^{2} / 2\left(\delta_{Q}^{2}+2 W^{2}\right)}\right],
\end{aligned}
$$

where $m_{Q}^{2}=c_{2}$.

\section{RESULTS}

We use the previously published $350 \mu \mathrm{m}$ SHARP polarization map of OMC-1 of Vaillancourt et al. (2008) to determine the turbulent correlation lengths of the Stokes $Q$ and $U$ parameters and the unpolarized emission $N$. Therefore, we refer to Equation (21) derived for the structure functions

$$
\begin{aligned}
\left\langle\Delta \bar{K}^{2}(\ell)\right\rangle= & m_{K}^{2} \ell^{2}+2 \sqrt{2 \pi}\left\langle K_{t}^{2}\right\rangle\left[\frac{\delta_{K}^{3}}{\left(\delta_{K}^{2}+2 W^{2}\right) \Delta}\right] \\
& \times\left[1-e^{-\ell^{2} / 2\left(\delta_{K}^{2}+2 W^{2}\right)}\right],
\end{aligned}
$$

in which $K$ stands for $Q, U$, or $N$. The coefficient $m_{K}^{2}$ belongs to the first-order term of the Taylor series and $\delta_{K}$ represents the turbulent correlation length scales of $Q, U$, and $N$. The calculations are done using the SHARP beam radius $W=4$ ".7 (or FWHM = 11"; see Houde et al. 2009).

Figures 1,2, and 3 show the results of our fits to the data for $Q, U$, and $N$, respectively. In all three figures, the data are shown with symbols. In the top graphs, the broken curve does not contain the correlated turbulent term of the function

$$
2 \sqrt{2 \pi}\left\langle K_{t}^{2}\right\rangle\left[\frac{\delta_{K}^{3}}{\left(\delta_{K}^{2}+2 W^{2}\right) \Delta}\right] e^{-\ell^{2} / 2\left(\delta_{K}^{2}+2 W^{2}\right)},
$$

but only includes the following two:

$$
m_{K}^{2} \ell^{2}+2 \sqrt{2 \pi}\left\langle K_{t}^{2}\right\rangle\left[\frac{\delta_{K}^{3}}{\left(\delta_{K}^{2}+2 W^{2}\right) \Delta}\right] .
$$

We fitted Equation (24) for values of $0.5 \lesssim \ell \lesssim 0.8$ (more details can be found in Appendix A of Houde et al. 2009). The intercept of the fit at $\ell=0$ shows the level of the turbulent component (i.e., the second term in the above equation) that can be compared to the first term, which depicts the contribution from the ordered part when $\ell \lesssim W$.

The middle plot shows the same information only plotted as a function of $\ell$. We subtract the data points from the fit of Equation (24) and show the results in bottom graphs (symbols). The correlated turbulent term (Equation (23)) is fitted to the 

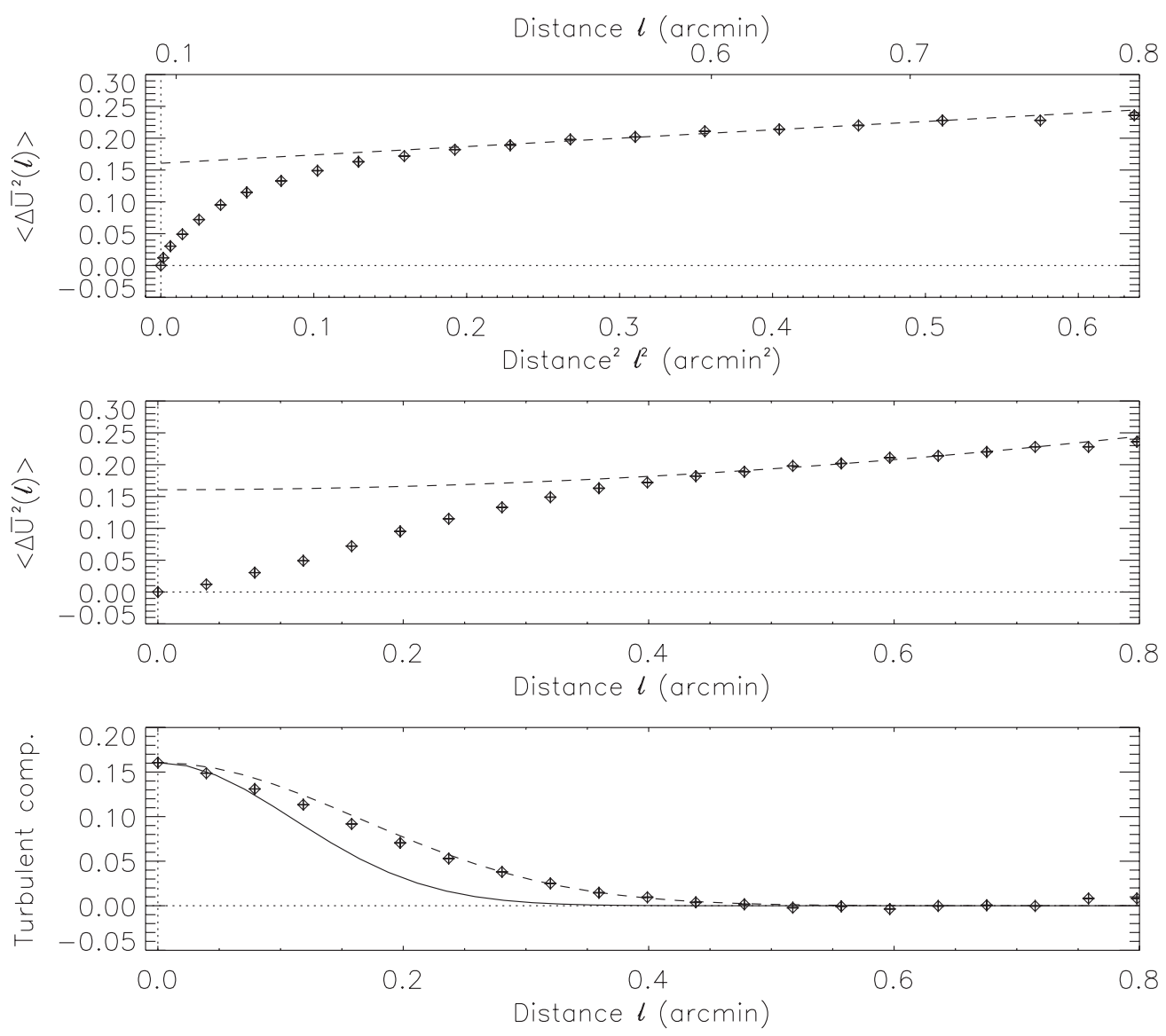

Figure 2. Same as Figure 1, but for $\left\langle\Delta \bar{U}^{2}(\ell)\right\rangle$. The turbulent correlation length is measured to be $\delta_{U}=7^{\prime \prime} .3 \pm 0^{\prime \prime}$. 1 .

Table 1

Results from our Fit of Equation (24) to the Dispersion Data for OMC-1

\begin{tabular}{lcc}
\hline \hline$\delta_{Q}$ & $\delta_{U}$ & $\delta_{N}$ \\
\hline $99^{\prime \prime} 4 \pm 0.1$ & $7^{\prime \prime} .3 \pm 0 ! 1$ & $12^{\prime \prime} .6 \pm 0.2$ \\
$20.5 \pm 0.2 \mathrm{mpc}$ & $16.0 \pm 0.2 \mathrm{mpc}$ & $27.5 \pm 0.4 \mathrm{mpc}$ \\
\hline
\end{tabular}

data (broken curve) with $\delta_{K}$ as the only fitting parameter to match the width of the function. Even though the autocorrelation functions of $Q, U$, and $N$ are assumed to have Gaussian patterns and the data points in the top and middle graphs in all three figures follow the fits quite well, it is quite unlikely that these are realistic models for these functions. The solid curve in the bottom graphs shows the contribution of the telescope beam alone (i.e., when $\delta_{K}=0$ in the exponent of Equation (23)).

The results from the fits are tabulated in Table 1 . We have measured the turbulent correlation length scales of Stokes $Q$ and $U$, and $N$ to be approximately $9 . .4 \pm 0.1,7^{\prime \prime} .3 \pm 0$. . 1 , and $12^{\prime \prime} .6 \pm 0.2$ (or $20.5 \pm 0.2,16.0 \pm 0.2$, and $27.5 \pm 0.4 \mathrm{mpc}$ at $450 \mathrm{pc}$, the adopted distance for OMC-1).

\section{DISCUSSION}

The study and analysis presented in this paper seek to characterize the magnetized turbulence in star-forming regions in molecular clouds through the determination of the turbulent correlation length scale $\delta$.

Dust grains are present in a variety of astronomical environments, such as molecular clouds, and also tend to couple to the magnetic field threading these regions mainly through their magnetic moment. Comparison of the turbulent correlation length scales of different components of the dust emission with the results from other techniques and methods is helpful in achieving a better understanding of turbulence, magnetic fields, and their interactions. The turbulent correlation length scales are evaluated from the autocorrelation function of the emission, which is the Fourier transform of the emission power spectrum, and therefore $\delta$ being inversely proportional to the width of the power spectrum yields valuable information. The turbulent correlation length scales of the Stokes parameters of the polarized emission $\left(\delta_{Q} \simeq 21 \mathrm{mpc}\right.$ and $\left.\delta_{U} \simeq 16 \mathrm{mpc}\right)$ are in agreement with that of the turbulent magnetic field ( $\delta \simeq 16 \mathrm{mpc}$ ) determined by Houde et al. (2009).

Another comparison can be made using the results presented in $\mathrm{Li} \&$ Houde (2008) for the turbulent power spectrum of coexisting ion and neutral molecular species in M17. The spectra for these two species share the same pattern in the inertial range, which is expected due to the tight coupling between ions and neutrals through flux freezing. However, their spectra cease to follow a common pattern as they decouple through turbulent ambipolar diffusion. The values determined for $\delta_{Q}$ and $\delta_{U}$ in this paper are consistent with the analysis presented in Houde et al. (2009), i.e., both $\delta_{Q}$ and $\delta_{U}$ are larger than the ambipolar diffusion scale, $\delta_{\mathrm{AD}}$, which was recently measured to be $9.9 \mathrm{mpc}$ in Orion KL by Houde et al. (2011). These measurements are also consistent with more general theoretical expectations related to turbulent ambipolar diffusion (Lazarian et al. 2004; McKee \& Ostriker 2004; Falceta-Gonçalves et al. 2010; Tilley \& Balsara 2010) and other observational measurements (Li \& Houde 2008; Hezareh et al. 2010). 

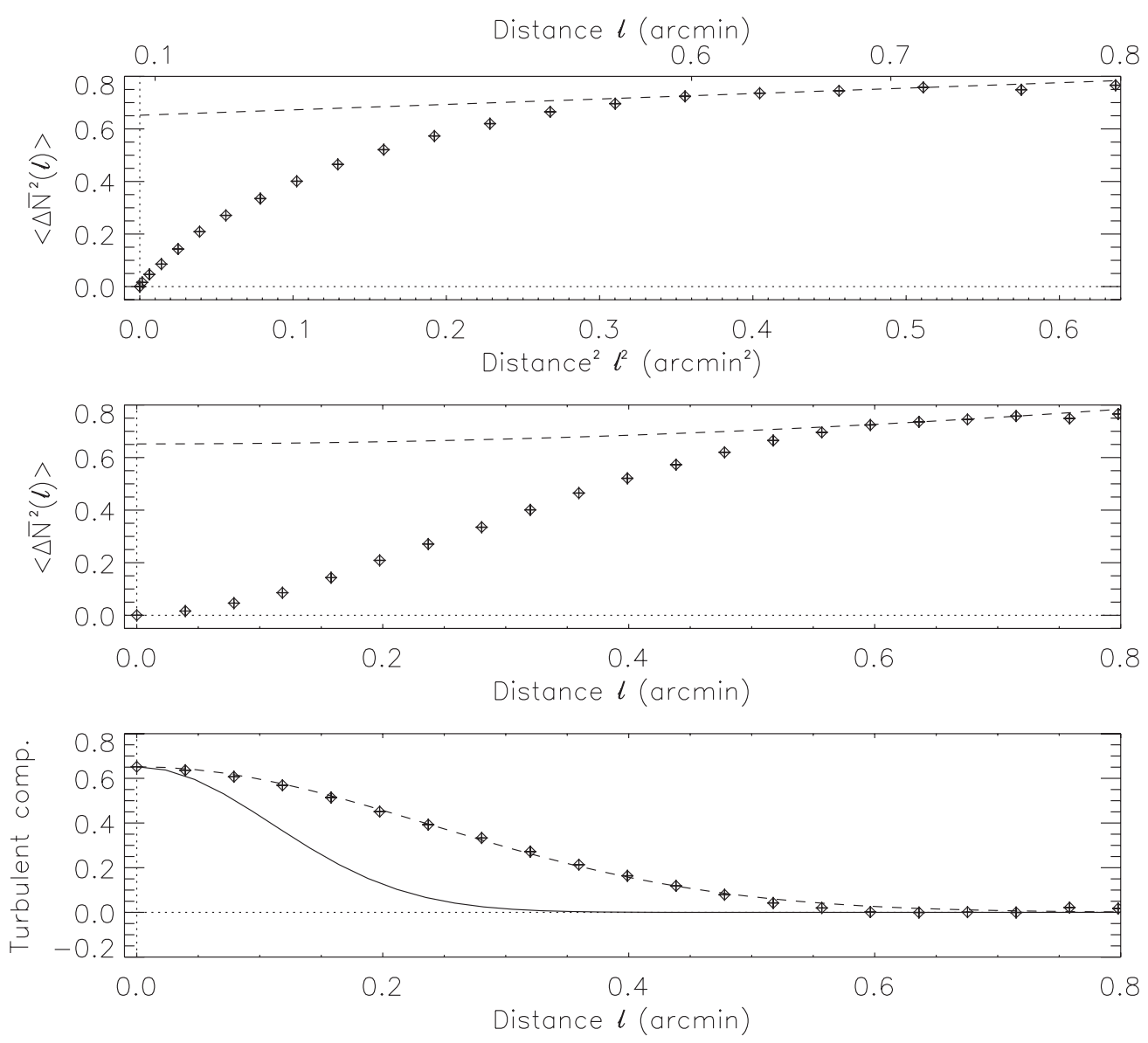

Figure 3. Same as Figure 1, but for $\left\langle\Delta \bar{N}^{2}(\ell)\right\rangle$. The turbulent correlation length is measured to be $\delta_{N}=12^{\prime \prime} .6 \pm 0$ '. 2 .

On the other hand, there is a measurable difference between the estimated value for $\delta_{N}(\simeq 28 \mathrm{mpc})$ and the correlation length scales of the Stokes parameters, i.e., $\delta_{Q}$ and $\delta_{U}$. This result implies that the power spectrum of $N$ is different from that of $Q$ and $U$. A lack or weakness of coupling between the magnetic field and the dust particles responsible for the unpolarized part of the emission can therefore be inferred from the results.

The Stokes parameters $Q$ and $U$ are each derived from simultaneous measurements of two orthogonal polarization states. Considering the uncertainties in our estimates of $\delta_{Q}$ and $\delta_{U}$, the difference between the values of these two parameters is significant and reveals the presence of anisotropy, which is expected for both incompressible (Goldreich \& Sridhar 1995; Cho et al. 2002) and compressible MHD turbulence (Cho \& Lazarian 2003; Kowal \& Lazarian 2010) and is consistent with the observational results of Heyer (2008). More precisely, it is predicted that the autocorrelation function of magnetized turbulence will have a longer length scale in a direction parallel to the magnetic field (as compared to an orientation perpendicular to the field). We have measured the mean polarization angle in OMC- 1 to be approximately $30^{\circ}$, as can be visually verified with the polarization map of this region presented in Figure 1(a) of Vaillancourt et al. (2008). This implies that the Stokes $U$ parameter will be dominated by the emission polarized (approximately) along the mean polarization vector and therefore perpendicular to the mean magnetic field. We thus expect that it will have a shorter correlation length scale compared to the Stokes $Q$ parameter, which will more or less be equally representative of emissions along and perpendicular to the magnetic field. This is consistent with our results, as presented in Table 1.

Obtaining a complete turbulent power spectrum is only possible through high-resolution observations and sufficient sampling in space, which are not available to us through polarization measurements with SHARP. Such analyses have, however, been recently conducted by Houde et al. (2011).

\section{CONCLUSIONS}

In this paper, we take advantage of the methods presented in Hildebrand et al. (2009) and Houde et al. (2009) to determine the turbulent structure functions of the Stokes parameters ( $Q$ and $U$ ) of the polarized emission and unpolarized emission $N$. Subsequently, the solutions are fitted to the previously published $350 \mu \mathrm{m}$ SHARP polarization map of OMC-1 of Vaillancourt et al. (2008) to estimate the turbulent correlation lengths of the Stokes parameters $\delta_{Q}$ and $\delta_{U}$ and the unpolarized emission $\delta_{N}$. Our results are consistent with the results presented in Houde et al. $(2009,2011)$ and may indicate presence of anisotropy in the magnetized turbulence. We also infer a weak coupling between the dust component responsible for the unpolarized emission $N$ and the magnetic field $\boldsymbol{B}$ from the significant difference between their turbulent correlation length scales.

M.H.'s research is funded through the NSERC Discovery Grant, Canada Research Chair, Canada Foundation for Innovation, Ontario Innovation Trust, and Western's Academic Development Fund programs. The CSO is funded through NSF AST 
05-40882. This work has also been supported in part by NSF grants AST 05-05230, AST 02-41356, and AST 05-05124.

\section{REFERENCES}

Attard, M., Houde, M., Novak, G., et al. 2009, ApJ, 702, 1584

Chandrasekhar, S., \& Fermi, E. 1953, ApJ, 118, 113

Cho, J., \& Lazarian, A. 2003, MNRAS, 345, 325

Cho, J., Lazarian, A., \& Vishniac, E. T. 2002, ApJ, 564, 291

Crutcher, R. M., Hakobian, N., \& Troland, T. H. 2010, MNRAS, 402, L64

Crutcher, R. M., Nutter, D. J., Ward-Thompson, D., \& Kirk, J. M. 2004, ApJ, 600,279

Curran, R. L., \& Chrysostomou, A. 2007, MNRAS, 382, 699

Elmegreen, B. G., \& Scalo, J. 2004, ARA\&A, 42, 211

Falceta-Gonçalves, D., Lazarian, A., \& Houde, M. 2010, ApJ, 713, 1376

Falceta-Gonçalves, D., Lazarian, A., \& Kowal, G. 2008, ApJ, 679, 537

Girart, J. M., Rao, R., \& Marrone, D. P. 2006, Science, 313, 812

Goldreich, P., \& Sridhar, S. 1995, ApJ, 438, 763

Heyer, M., Gong, H., Ostriker, E., \& Brunt, C. 2008, ApJ, 680, 420

Hezareh, T., Houde, M., McCoey, C., \& Li, H. 2010, ApJ, 720, 603

Hildebrand, R. H., Kirby, L., Dotson, J. L., Houde, M., \& Vaillancourt, J. E. 2009, ApJ, 696, 567
Houde, M. 2004, ApJ, 616, 111

Houde, M., Rao, R., Vaillancourt, J. E., \& Hildebrand, R. H. 2011, ApJ, 733, 109

Houde, M., Vaillancourt, J. E., Hildebrand, R. H., Chitsazzadeh, S., \& Kirby, L. 2009, ApJ, 706, 1504

Kowal, G., \& Lazarian, A. 2010, ApJ, 720, 742

Lai, S.-P., Crutcher, R. M., Girart, J. M., \& Rao, R. 2001, ApJ, 561, 864

Lai, S.-P., Crutcher, R. M., Girart, J. M., \& Rao, R. 2002, ApJ, 566, 925

Lai, S.-P., Girart, J. M., \& Crutcher, R. M. 2003, ApJ, 598, 392

Lazarian, A., Vishniac, E. T., \& Cho, J. 2004, ApJ, 603, 180

Li, H., \& Houde, M. 2008, ApJ, 677, 1151

Mac Low, M., \& Klessen, R. S. 2004, Rev. Mod. Phys., 76, 125

McKee, C. F., \& Ostriker, E. C. 2007, ARA\&A, 45, 565

Mouschovias, T. Ch., \& Ciolek, G. E. 1999, in The Origin of Stars and Planetary Systems, ed. C. J. Lada \& N. D. Kylafis (Dordrecht: Kluwer), 305

Mouschovias, T. Ch., \& Tassis, K. 2009, MNRAS, 400, L15

Nakano, T. 1984, Fundam. Cosm. Phys., 9, 139

Shu, F. H., Allen, A., Shang, H., Ostriker, E. C., \& Li, Z. 1999, in The Origin of Stars and Planetary Systems, ed. C. J. Lada \& N. D. Kylafis (Dordrecht: Kluwer), 193

Tilley, D. A., \& Balsara, D. S. 2010, MNRAS, 406, 1201

Vaillancourt, J. E., Dowell, D. C., Hildebrand, R. H., et al. 2008, ApJ, 679, 25 